\title{
Psychotic symptoms are associated with lower cortical folding in youth at risk for mental illness
}

\author{
Vladislav Drobinin, BSc; Holly Van Gestel, MSc; Alyson Zwicker, PhD; Lynn MacKenzie, PhD; \\ Jill Cumby, MN; Victoria C. Patterson, BA; Emily Howes Vallis, MSc; Niamh Campbell, BSc; \\ Tomas Hajek, MD, PhD; Carl A. Helmick, BSC; Matthias H. Schmidt, MSc, MD; \\ Martin Alda, MD; Chris V. Bowen, PhD; Rudolf Uher, MD, PhD
}

\begin{abstract}
Background: Cortical folding is essential for healthy brain development. Previous studies have found regional reductions in cortical folding in adult patients with psychotic illness. It is unknown whether these neuroanatomical markers are present in youth with subclinical psychotic symptoms. Methods: We collected MRIs and examined the local gyrification index in a sample of 110 youth (mean age \pm standard deviation $14.0 \pm 3.7 \mathrm{yr}$; range 9-25 yr) with a family history of severe mental illness: 48 with psychotic symptoms and 62 without. Images were processed using the Human Connectome Pipeline and FreeSurfer. We tested for group differences in local gyrification index using mixed-effects generalized linear models controlling for age, sex and familial clustering. Sensitivity analysis further controlled for intracranial volume, IQ, and stimulant and cannabis use. Results: Youth with psychotic symptoms displayed an overall trend toward lower cortical folding across all brain regions. After adjusting for multiple comparisons and confounders, regional reductions were localized to the frontal and occipital lobes. Specifically, the medial $\left(B=-0.42, p_{F D R}=0.04\right)$ and lateral $\left(B=-0.39, p_{F D R}=0.04\right)$ orbitofrontal cortices as well as the cuneus $\left(B=-0.47, p_{F D R}=0.03\right)$ and the pericalcarine $\left(B=-0.45, p_{F D R}=0.03\right)$ and lingual $(B=-0.38$, $\left.p_{F D R}=0.04\right)$ gyri. Limitations: Inference about developmental trajectories was limited by the cross-sectional data. Conclusion: Psychotic symptoms in youth are associated with cortical folding deficits, even in the absence of psychotic illness. The current study helps clarify the neurodevelopmental basis of psychosis at an early stage, before medication, drug use and other confounds have had a persistent effect on the brain.
\end{abstract}

\section{Introduction}

Psychosis is marked by hallucinations, delusions and disturbances of affect and behaviour. It manifests in major depressive disorder, bipolar disorder and most notably, schizophrenia. Although psychotic disorders are a leading cause of morbidity and disability worldwide, ${ }^{1}$ their etiology remains unclear. ${ }^{2}$ Multiple lines of evidence are converging to suggest that the disease stems from processes that affect neurodevelopment. ${ }^{3}$ The neurodevelopmental hypothesis posits that genetic and environmental risk factors perturb early brain development, leading to symptoms later in life as the brain matures and copes with new stressors. ${ }^{4}$ One process necessary for healthy brain development is cortical folding, which may be abnormal in the pathogenesis of schizophrenia. ${ }^{5}$

The process of cortical folding, or gyrification, results in gyri and sulci that give the cortex its wrinkly appearance. The degree of cortical folding can be quantified using the local gyrification index (LGI). ${ }^{6}$ The LGI is a ratio of the total cortical surface to the superficially exposed outer surface tightly wrapping the cortex without entering the sulci. Cortical folding is a uniquely mammalian solution to increasing cortical grey matter without exaggerating head size. This process is also key to the optimization of axonal wiring and the functional organization of the brain. ${ }^{7}$ The mechanisms of cortical folding are under active investigation. Recent perspectives suggest that tightly coordinated molecular genetic processes $^{8}$ and biomechanical forces ${ }^{9}$ are involved. Radial expansion of progenitor cells might be particularly significant (see Fernández and colleagues ${ }^{10}$ for a review). Importantly, cortical folding provides a window on early development. ${ }^{11}$ The major folding patterns are determined largely before birth and finish undergoing the most rapid morphological changes by childhood. ${ }^{12}$ This sensitive period of neurodevelopment overlaps with the timing of the most prominent environmental risk factors associated with psychosis. ${ }^{13}$

Large multisite neuroimaging studies have found reductions in cortical folding among adults with psychotic disorders. ${ }^{14,15}$ Aberrant gyrification has also been reported in people at genetic risk for schizophrenia. ${ }^{14,16}$ By extension, recent work

Correspondence to: V. Drobinin, 13E, 5850 College St., Halifax, NS, B3H 4H7; vlad.drobinin@ dal.ca

Submitted Aug. 21, 2018; Revised Jun. 17, 2019; Accepted Jul. 3, 2019; Published online Nov. 1, 2019

DOI: 10.1503/jpn.180144 
has shown that scores indexing genetic liability to schizophrenia are associated with regional reductions in the LGI. ${ }^{17}$ The prefrontal cortex has long been implicated in schizophrenia. ${ }^{18}$ Early work that measured cortical folding with manual tracing of MRI slices has shown lower frontal cortical folding in patients with schizophrenia. ${ }^{19}$ Correspondingly, recent work using automated 3D methods to quantify LGI have shown similar reductions in prefrontal cortical folding. ${ }^{20}$

The reported prefrontal LGI abnormalities are consistent with neuropathological findings from other imaging modalities and postmortem data. ${ }^{21,22}$ Abnormal gyrification also predicts poor treatment response in first-episode psychosis ${ }^{23}$ and has been used to distinguish patients with more severe illness from those with milder forms. ${ }^{24}$ Taken together, the body of literature suggests cortical folding alterations across the psychosis spectrum. However, whether or not these alterations are present before illness onset has been more difficult to establish.

Adolescence has been described as a critical period of vulnerability for schizophrenia. ${ }^{4}$ However, the majority of clinical high-risk studies have focused on adulthood. ${ }^{25}$ As such, the etiology of psychosis can be clarified by examining cortical folding earlier, before the onset of a functionally impairing illness. In the current study, we addressed this gap by examining the LGI in adolescents from a cohort with enriched familial risk who had experienced psychotic symptoms but did not meet the criteria for psychotic illness. We hypothesized that psychotic symptoms would be related to lower cortical folding in symptomatic youth, particularly in the prefrontal cortex.

\section{Methods}

\section{Participants}

As part of the ongoing Families Overcoming Risks and Building Opportunities for Well-being (FORBOW) study, we collected MRI scans from 110 participants aged 9-25 years. The FORBOW study is a longitudinal study enriched for the offspring of parents with mental illness. ${ }^{26}$ Those at familial risk for mental illness and participants from control families were invited to complete the MRI study. The study protocol was approved by the research ethics board of the Nova Scotia Health Authority. Participants provided written informed consent. For children who did not have the capacity to make a fully informed decision, a parent or guardian provided written informed consent and the child provided assent. Exclusion criteria were a personal history of psychotic illness, any serious medical or neurologic disorder, substance abuse or dependence during the previous 6 months, or MRI contraindications.

\section{Participant clinical and cognitive assessment}

\section{Parent assessment}

We used the Schedule for Affective Disorders and Schizophrenia (SADS-IV) ${ }^{27}$ and the Structured Clinical Interview for DSM-5 (SCID-5) ${ }^{28}$ to establish diagnoses of mental disorders and psychosis according to DSM-IV and DSM-5. Diagnoses were confirmed in consensus meetings with a psychiatrist blind to offspring psychopathology.

\section{Offspring assessment}

Participating youth were interviewed using the Kiddie Schedule for Affective Disorders and Schizophrenia, Present and Lifetime Version (K-SADS-PL). ${ }^{29}$ Offspring assessors were blinded to parent psychopathology. Full-scale intelligence quotient (FSIQ) was assessed using the Wechsler Abbreviated Scale of Intelligence, second edition. ${ }^{30}$ Psychotic symptoms were assessed using the following instruments: the K-SADS-PL interview psychosis module and appendix, consensus-rated by child and adolescent psychiatrists blind to parent psychopathology; in participants aged 3-12 years, the Structured Interview for Prodromal Syndromes (SIPS), ${ }^{31}$ measuring attenuated psychotic symptoms; the "Funny Feelings" interview ${ }^{32-34}$ (we included only youth with symptoms rated as "definite psychotic symptom" by consensus between 2 independent raters); the Schizophrenia Proneness Instrument, Child and Youth version (SPI-CY) ${ }^{35}$ for those aged 8 years and older, to assess abnormal experiences in the domains of perception, cognition, language and affect that strongly predict the development of psychosis. ${ }^{36,37}$

Consistent with our previous reports, ${ }^{38,39}$ we created a dichotomous variable for the presence of psychotic symptoms captured by any one of the following: confirmed hallucinations or delusions on K-SADS-PL, positive symptoms on SIPS rated $\geq 3$, definite psychotic symptoms confirmed through independent curation, and high-risk basic symptom profiles of cognitive/perceptive disturbances on the SPI-CY.

\section{MRI acquisition}

Images were acquired with a 3 T General Electric Discovery MR750 scanner equipped with a 32-channel MR Instruments radiofrequency head coil. Scanning took place at the Biomedical Translational Imaging Centre (BIOTIC) in Halifax, Nova Scotia, Canada. Each participant was positioned supine in the MRI scanner, with their head supported by foam padding to reduce movement. Ear plugs were provided to minimize scanner noise. We collected a 3D $T_{1}$-weighted brain volume imaging (BRAVO) sequence with whole-brain coverage; $1 \mathrm{~mm}^{3}$ isotropic resolution; matrix $224 \times 224$; field of view $224 \mathrm{~mm}$; 168 sagittal slices at $1 \mathrm{~mm}$ thickness; repetition time $5.9 \mathrm{~ms}$; echo time $2.2 \mathrm{~ms}$; inversion time $450 \mathrm{~ms}$; flip angle $12^{\circ}$; receiver bandwidth \pm 62.5 ; number of excitations $=2$; autocalibrating reconstruction for cartesian imaging (ARC) phase acceleration $=2$; ARC slice acceleration $=1$; no phase wrap; scan duration 5 minutes, 42 seconds.

\section{MRI analysis}

Data were preprocessed using the open-source Human Connectome Pipeline. ${ }^{40}$ As part of the validated pipeline, we reconstructed the $T_{1}$-weighted scan surface using FreeSurfer version 5.3. ${ }^{41}$ An automated labelling system subdivided the cerebral cortex into gyral-based parcellations corresponding to the Desikan-Killiany atlas. ${ }^{42}$ 


\section{Local gyrification index}

We quantified the 3D LGI from FreeSurfer output, as the ratio of the total cortical surface area (including cortex buried in sulci) to the outer cortex surface area, which tightly wraps the brain but does not enter the sulci. Thus, a higher LGI represents more cortical folding in each brain parcel, and a lower LGI represents less cortical folding. Details of the automated LGI computation can be found in the validation paper $^{6}$ and on the FreeSurfer website (https://surfer.nmr.mgh.harvard. edu/fswiki/LGI).

\section{Statistical analysis}

We performed statistical analyses in $\mathrm{R}$ Studio (version 3.5.0). ${ }^{43}$ We compared demographic and clinical variables using $t$ tests for continuous variables and $\chi^{2}$ tests for categorical variables. The LGI for each of the 34 cortical parcellations across both hemispheres served as the primary dependent variable. The primary independent variable was the presence or absence of psychotic symptoms.

We tested the relationship between lifetime psychotic symptoms and LGI using mixed-effect generalized linear models. We accounted for the non-independence of brain data from related individuals by including the family identifier as a random effect. We included age and sex as covariates in the model. We controlled for multiple comparisons across brain parcellations using false discovery rate (FDR). ${ }^{44}$ We reported effect sizes using standardized regression estimates (B) and their $95 \%$ confidence intervals (CIs).

To ensure that the observed relationship between cortical folding and psychotic symptoms was not due to the use of psychoactive substances or other factors linked to changes in brain structure, we conducted sensitivity analyses. In the brain regions found to be significant after correcting for multiple testing, we further covaried for lifetime cannabis use, lifetime stimulant use, FSIQ and estimated total intracranial volume (eTIV).

\section{Results}

\section{Demographic variables}

Of the 110 youth scanned, 48 (43.64\%) met the criteria for a definite psychotic symptom on 1 or more assessments. Table 1 summarizes demographic and clinical characteristics by symptom status. General cognitive ability did not differ between participants with or without psychotic symptoms $(t=$ $0.67, p=0.51)$. We found a statistically significant difference in eTIV $(t=-3.15, p=0.002)$ : youth with psychotic symptoms showed smaller eTIV (mean $1427.8 \pm 168.0 \mathrm{~cm}^{3}$ ) than youth without symptoms $\left(1522.2 \pm 138.6 \mathrm{~cm}^{3}\right)$.

\section{Overall differences in cortical folding across brain regions}

We examined overall differences in cortical folding averaged across all brain regions. In a model controlling for sex, age and familial clustering, average whole-brain cortical folding was lower in youth with psychotic symptoms $(B=-0.13,95 \%$ CI -0.21 to $-0.05, p=0.001)$. This effect was no longer statistically significant following covariance for eTIV $(B=-0.05$, $95 \% \mathrm{CI}-0.13$ to $0.03, p=0.22$ ). Figure 1 shows the mean differences in folding across all structures.

\section{Prefrontal cortical folding}

We used the same model to explore regional differences in cortical folding. In line with our hypothesis, the exploratory analysis revealed lower prefrontal cortical folding in youth with psychotic symptoms (Fig. 2). Specifically, the mixedeffect generalized linear models controlling for sex, age and familial clustering localized the lower cortical folding to the medial $\left(B=-0.42,95 \% \mathrm{CI}-0.71\right.$ to $-0.12, p=0.006, p_{\mathrm{FDR}}=$ $0.04)$ and lateral $(B=-0.39,95 \% \mathrm{CI}-0.66$ to $-0.12, p=0.005$, $\left.p_{\mathrm{FDR}}=0.04\right)$ aspects of the orbitofrontal cortex $(\mathrm{OFC})$.

\begin{tabular}{lccc} 
Table 1: Demographic and clinical characteristics of the study sample $(\boldsymbol{n}=\mathbf{1 1 0})^{*}$ & \\
\hline Characteristic & No psychotic symptoms & Psychotic symptoms & $p$ value \\
\hline Participants, no. & 62 & 48 & 0.08 \\
Female sex, no. (\%) & $31(50.0)$ & $33(68.8)$ & 0.28 \\
Mean age \pm SD, yr & $13.7 \pm 3.4$ & $14.5 \pm 4.1$ & 0.49 \\
Siblings, no. (\%) & $11(17.8)$ & $12(25.0)$ & 0.09 \\
Anxiety disorder, no. (\%) & $21(33.9)$ & $25(52.1)$ & 0.12 \\
Parent diagnosis, no. (\%) & & & - \\
None & $18(29.0)$ & $6(12.5)$ & - \\
Depression & $23(37.1)$ & $27(56.3)$ & - \\
Bipolar disorder & $17(27.4)$ & $13(27.1)$ & - \\
Schizophrenia & $4(6.5)$ & $9(4.2)$ & 1.00 \\
Parent psychosis, no. (\%) & $11(17.8)$ & $6(12.5)$ & 1.00 \\
Cannabis use, no. (\%) & $7(11.3)$ & $6(12.5)$ & 0.08 \\
Stimulant use, no. (\%) & $2(3.2)$ & $107.9 \pm 11.3$ & 0.51 \\
Mean FSIQ score \pm SD & $106.2 \pm 14.3$ & $1427.8 \pm 168.0$ & 0.002 \\
Mean eTIV \pm SD, cm ${ }^{3}$ & $1522.2 \pm 138.6$ & & - \\
\hline eTIV = estimated total intracranial volume; FSIQ $=$ Full Scale Intelligence Quotient; SD standard deviation. \\
*We compared demographic and clinical variables using $t$ tests for continuous variables and $\chi^{2}$ tests for \\
categorical variables. & & & \\
\hline
\end{tabular}


In sensitivity analyses of this finding, youth with psychotic symptoms robustly showed lower OFC folding in models controlling for cannabis use, stimulant use, FSIQ and eTIV, both medially ( $B=-0.34,95 \% \mathrm{CI}-0.63$ to $-0.04, p=0.025)$ and laterally $(B=-0.28,95 \% \mathrm{CI}-0.54$ to $-0.01, p=0.042)$.

\section{Occipital lobe cortical folding}

Along with the OFC findings, the exploratory analysis revealed 3 additional regions that survived brain-wide correction for multiple comparisons in models controlling for age,

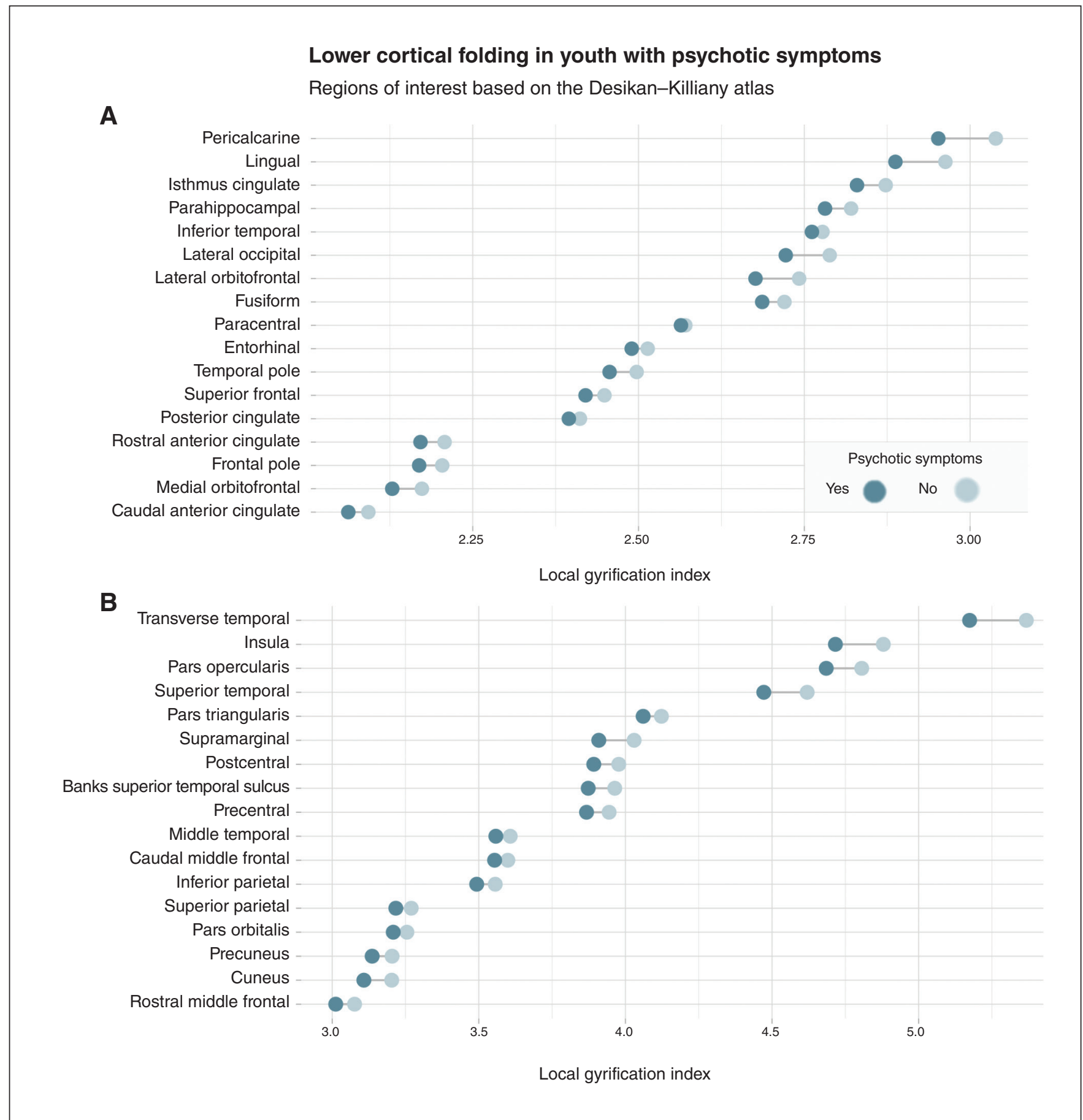

Fig. 1: Differences in mean cortical folding across the anatomical boundaries of the Desikan-Killiany atlas. Dark blue dots represent youth with psychotic symptoms $(n=48)$, light blue dots represent those without $(n=62)$. We found an overall trend toward lower cortical folding in youth with psychotic symptoms. (A) Regions of interest with mean local gyrification index $<3.0$. (B) Regions of interest with mean local gyrification index $>3.0$. 
sex and familial clustering (Fig. 3). Psychotic symptoms were associated with lower regional gyrification in the occipital lobe-specifically the cuneus $(B=-0.47,95 \% \mathrm{CI}-0.75$ to $\left.-0.19, p=0.001, p_{\mathrm{FDR}}=0.03\right)$, the pericalcarine gyrus $(B=$ $-0.45,95 \% \mathrm{CI}-0.73$ to $\left.-0.17, p=0.002, p_{\mathrm{FDR}}=0.03\right)$ and the lingual gyrus $\left(B=-0.38,95 \% \mathrm{CI}-0.66\right.$ to $-0.11, p=0.006, p_{\mathrm{FDR}}=$ $0.04)$. We saw the same pattern of results when examining symptoms from a dimensional perspective (Appendix 1, available at jpn.ca/180144-a1).

We conducted sensitivity analyses to determine if the differences in cuneus, pericalcarine and lingual cortical folding might be attributable to extraneous variables rather than to symptom status. Again, we implemented models identical to the exploratory analysis while simultaneously covarying for cannabis use, stimulant use, FSIQ and eTIV. As with the prefrontal results, the link between psychotic symptoms and occipital folding remained significant for the cuneus $(B=$ $-0.46,95 \% \mathrm{CI}-0.74$ to $-0.18, p=0.001)$ and the pericalcarine $(B=-0.40,95 \% C I-0.69$ to $-0.11, p=0.007)$ and lingual $(B=$ $-0.35,95 \%$ CI -0.63 to $-0.07, p=0.015)$ gyri.

\section{Discussion}

We sought to determine whether youth with psychotic symptoms displayed cortical aberrations before the onset of impairing psychotic illness. To answer that question, we examined 3D reconstructions of cortical folding, an early neurodevelopmental marker of cortical expansion. We found a pattern of lower cortical folding in adolescents who had psychotic symptoms but who did not meet the criteria for a psychotic disorder.

\section{Lower cortical folding}

In our study, psychotic symptoms were related to lower cortical folding across all brain regions, with statistically significant regional effects. This unidirectional pattern is supported by the literature on brain structure among adult patients with schizophrenia-spectrum disorders. Nesvåg and colleagues ${ }^{15}$ examined cortical folding among 207 patients with schizophrenia and 206 controls. They found that patients had a lower LGI in large clusters of the cerebral cortex, leading them to conclude that reduced gyrification is a feature of the brain pathology in schizophrenia. Similar to our work, no regions had significantly higher LGI among patients. Nanda and colleagues ${ }^{14}$ examined the LGI in 388 patients with psychotic disorders and 243 controls. Their multicentre study found that patients with psychotic disorders had a significantly lower LGI than controls. Importantly, the directionality of the finding was consistent with our findings, in that no regions were less folded among controls.

\section{Differences in prefrontal cortex folding}

Lower prefrontal cortical folding was localized to the medial and lateral OFC. Our finding was in line with previous work implicating abnormalities in the OFC cortical folding pattern in psychosis, ${ }^{23,45}$ first-episode schizophrenia ${ }^{46}$ and chronic

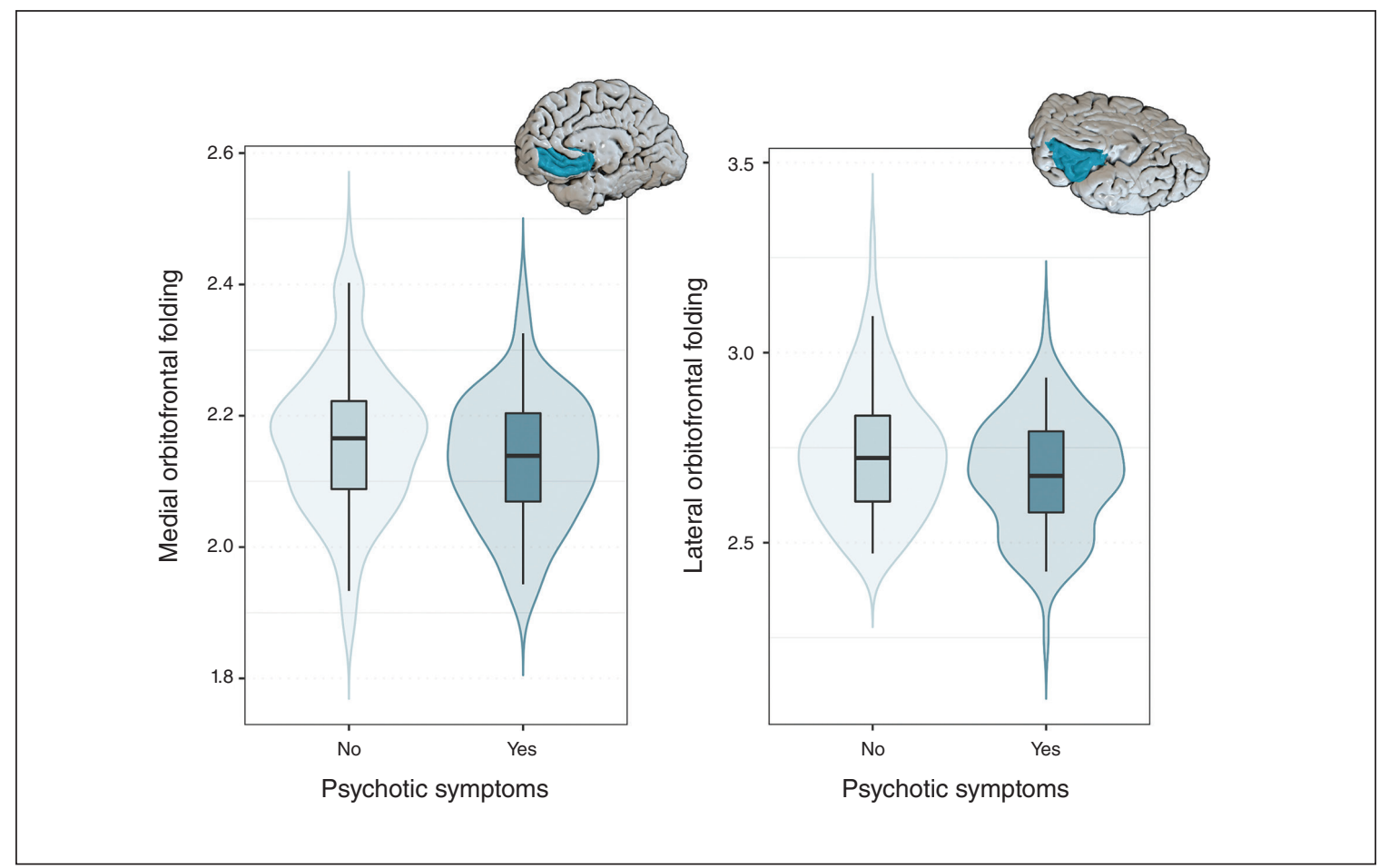

Fig. 2: Lower prefrontal cortical folding in youth with psychotic symptoms $(n=48)$ versus those without $(n=62)$. Symptomatic youth in darker blue. Violin plots present cortical folding distributions by symptom status. Box plots nested within display median differences. 


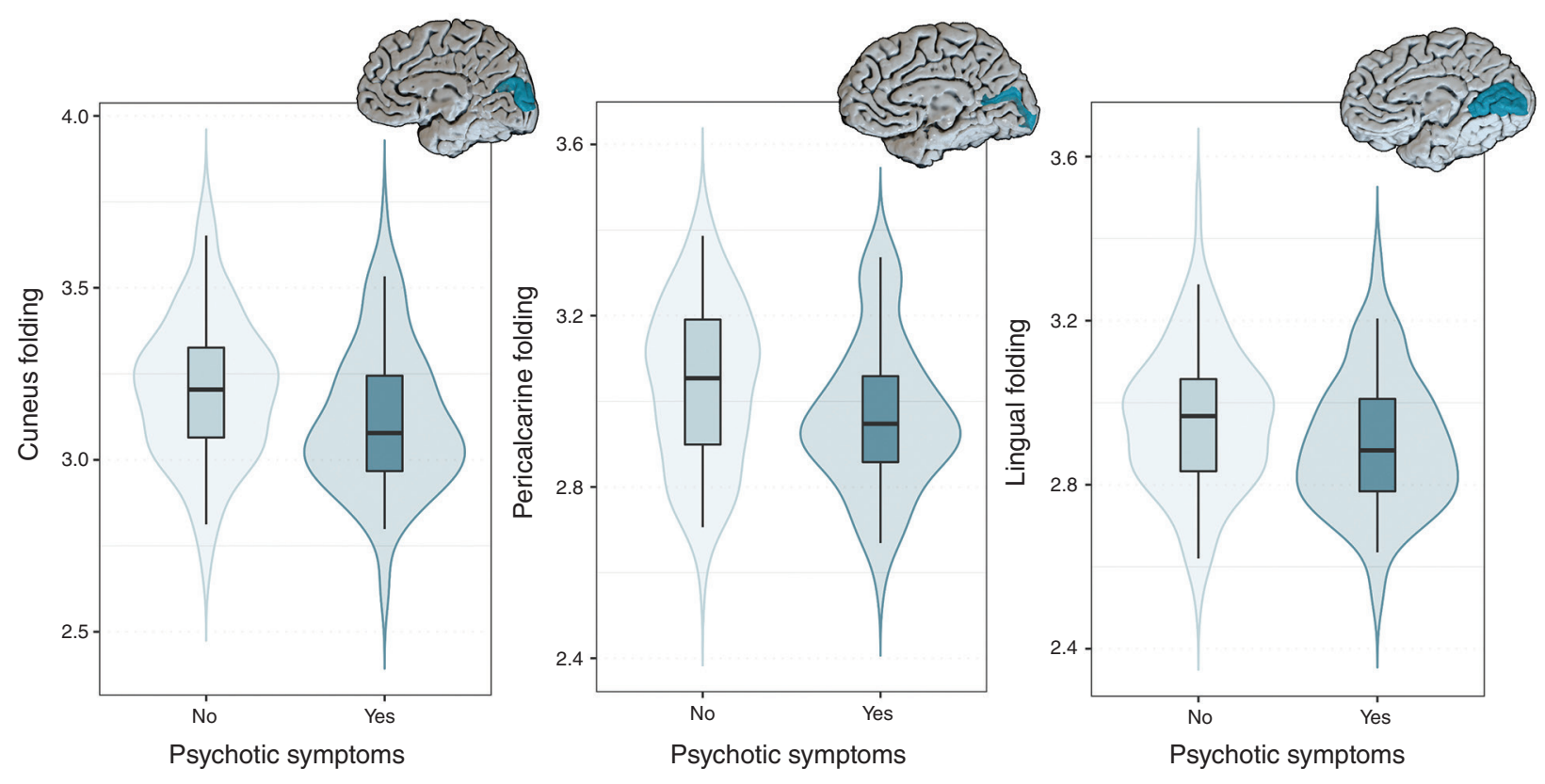

Fig. 3: Lower occipital cortical folding in youth with psychotic symptoms $(n=48)$ versus those without $(n=62)$. Symptomatic youth in darker blue. Violin plots present cortical folding distributions by symptom status. Box plots nested within display median differences.

schizophrenia. ${ }^{47}$ In our adolescent sample, this difference was unlikely to be attributable to common confounders in psychosis research, such as illness burden or the effects of medication. The localization to the OFC in our study was consistent with prospective studies in patients at clinical high risk and in animal models. Patients at clinical high risk who convert to psychosis show steeper rates of cortical thickness decline and grey matter reduction in the OFC than nonconverters at clinical high risk and controls. ${ }^{48,49}$ Furthermore, OFC neurons have been shown to be common targets for both typical and atypical antipsychotic drugs. ${ }^{50}$

Characteristics of schizophrenia include aberrant perception and cognitive deficits. The OFC is involved in a number of disorder-related functions, such as sensory integration, learning, and social and emotional decision-making. ${ }^{51,52}$ Our previous work examining cognition showed that youth with psychotic symptoms exhibited deficits in executive functioning. ${ }^{39}$ We specifically found impaired emotional decisionmaking, even after controlling for general cognitive ability. The combination of a reduction in OFC folding and impaired decision-making may be a neurocognitive marker of a propensity for psychotic symptoms.

\section{Differences in occipital cortex folding}

We found lower cortical folding in the cuneus and the pericalcarine and lingual gyri. The cuneus is located between the calcarine and parieto-occipital sulci. The pericalcarine gyrus can be visualized between the cuneus and lingual gyrus on a midline view of the brain. The lingual gyrus sits within the tentorial surface of the occipital lobe, inferior to the calcarine sulcus.

Studies of cortical folding and functional connectivity in psychosis have found reduced cortical folding in the lingual cortex of patients with psychosis compared with healthy controls. ${ }^{23,53}$ They also showed that the aberrant functional connectivity of the visual processing regions was a better predictor of symptom persistence and burden than diagnostic information. Our current work and aforementioned findings also correspond to studies examining volumetric data. Compared to healthy controls, patients with first-episode schizophrenia demonstrated significantly reduced grey matter volumes in the lingual gyrus. ${ }^{54}$ Significant cortical thinning has also been noted in this region in patients diagnosed with schizophrenia compared to matched controls. ${ }^{55,56}$ Finally, 22q11 deletion syndrome and genetic risk for schizophrenia based on common genetic variation have been associated with volumetric grey matter differences in the lingual gyrus and cuneus, as well as cortical thinning in the cuneus and pericalcarine and lingual gyri. ${ }^{57,58}$

A recent paper examined LGI in medicated young adults who met the criteria for an at-risk mental state. ${ }^{59}$ In contrast to our findings of decreased LGI, those in an at-risk mental state showed widespread increases in LGI. Interestingly, increased gyrification in the cuneus, pericalcarine 
and lingual regions was related to risk for transition to a psychotic disorder. This work underscores the relevance of occipital cortical folding in the risk for psychosis, but the discrepancy in the directionality of findings indicates the need for further longitudinal study of the developmental transition period from adolescence to young adulthood in high-risk populations.

\section{Limitations}

This study had several limitations. The onset of schizophrenia and other psychotic disorders typically occurs in late adolescence or early adulthood. In our developmental sample, the age range overlapped with this period. In other words, our sample included participants younger than the age with the highest risk of onset, and participants passing through this stage. Future studies can reduce this heterogeneity, particularly in cohorts examining brain development at fixed age ranges. Furthermore, the cross-sectional nature of this work limited our ability to track prospective developmental changes in regions with reduced folding. To address some of these challenges, we propose the collection of longitudinal imaging follow-up data.

We were able to minimize confounds of illness burden, comorbidities and medication use by studying youth. Nevertheless, a proportion of the sample had been exposed to marijuana and psychoactive medication, both of which could have an effect on the developing brain. ${ }^{60,61}$ Sensitivity analyses suggested that these substances did not affect the association between reduced cortical folding and psychotic symptoms. Cannabis use remains an important covariate to control for, because adolescent initiation of cannabis use has been linked to early-onset psychosis, ${ }^{62}$ and legalization of the substance may affect initiation or usage.

We found a significant difference in total intracranial volume in our sample. Youth with psychotic symptoms had lower eTIV. Although this finding was not part of our hypothesis, there is meta-analytic evidence for reduced intracranial and total brain volume in schizophrenia. ${ }^{63}$ Because certain structures scale with intracranial volume, ${ }^{64}$ we controlled for eTIV as a covariate. This correction eliminated the overall average difference in cortical folding across the brain, but the regional differences in frontal and occipital cortical folding remained robust to this correction. Future work is needed to contextualize the clinical relevance of eTIV differences in samples of risk-enriched youth.

Finally, we examined a single neurodevelopmental marker, but we know that cortical folding is related to optimization of axonal wiring and functional organization in the brain. ${ }^{7}$ Future research should integrate additional imaging data, such as probabilistic tractography, intracortical myelination and resting-state functional connectivity. For example, one multi-analysis study showed that decreased frontal gyrification in adolescent schizophrenia may be associated with widening of the frontal sulci and reductions in cortical surface area. ${ }^{65}$ Multimodal extension and synthesis with molecular genetic and neurocognitive data will bring new insights into the significance of our findings.

\section{Conclusion}

This study found regional reductions in cortical folding of adolescents who had experienced psychotic symptoms. The young age of the cohort helped to clarify the neurodevelopmental basis of psychosis at an early stage, before medication, drug use and illness burden could take a persistent toll on the brain. Our findings also suggest that measurement of cortical folding may play a role in the early detection of people at risk for psychotic illness.

Acknowledgements: This project was supported by the Independent Investigator Award, Brain \& Behavior Research Foundation (R. Uher; grant number 24684), the Canada Research Chairs Program (award number 231397), the Canadian Institutes of Health Research (grant reference numbers 124976, 142738 and 148394), the Nova Scotia Health Authority and the Dalhousie Medical Research Foundation. V. Drobinin was supported by the Canadian Institutes of Health Research doctoral award (157975). The Biomedical Translational Imaging Centre (BIOTIC) has received funding support from Brain Canada.

Affiliations: From the Department of Medical Neuroscience, Dalhousie University, Halifax, NS, Canada (Drobinin, Schmidt, Uher); the Nova Scotia Health Authority, Halifax, NS (Drobinin, van Gestel, Zwicker, MacKenzie, Cumby, Patterson, Vallis, Campbell, Helmick, Alda, Bowen, Uher); the Department of Pathology, Dalhousie University, Halifax, NS (Zwicker, Uher); the Department of Psychology and Neuroscience, Dalhousie University, Halifax, NS (MacKenzie, Patterson, Uher); the Department of Psychiatry, Dalhousie University, Halifax, NS (Vallis, Helmick, Alda, Uher); the Department of Medicine, Dalhousie University, Halifax, NS (Campbell); and the Department of Diagnostic Radiology, Dalhousie University, Halifax, NS (Bowen).

\section{Competing interests: None declared.}

Contributors: V. Drobinin, M. Schmidt, M. Alda, C. Bowen and R. Uher designed the study. V. Drobinin, H. van Gestel, A. Zwicker, L. MacKenzie, J. Cumby, V. Patterson, E. Howes Vallis, N. Campbell, T. Hajek, C. Helmick and R. Uher acquired the data, which V. Drobinin and R. Uher analyzed. V. Drobinin wrote the article, which all authors reviewed. All authors approved the final version to be published and can certify that no other individuals not listed as authors have made substantial contributions to the paper.

\section{References}

1. Global Burden of Disease Study 2013 Collaborators. Global, regional, and national incidence, prevalence, and years lived with disability for 301 acute and chronic diseases and injuries in 188 countries, 1990-2013: a systematic analysis for the Global Burden of Disease Study 2013. Lancet 2015;386:743-800.

2. Radua J, Ramella-Cravaro V, Ioannidis JPA, et al. What causes psychosis? An umbrella review of risk and protective factors. World Psychiatry 2018;17:49-66.

3. Rapoport JL, Giedd JN, Gogtay N. Neurodevelopmental model of schizophrenia: update 2012. Mol Psychiatry 2012;17:1228-38.

4. Selemon LD, Zecevic N. Schizophrenia: a tale of two critical periods for prefrontal cortical development. Transl Psychiatry 2015;5:e623.

5. White T, Gottesman I. Brain connectivity and gyrification as endophenotypes for schizophrenia: weight of the evidence. Curr Top Med Chem 2012;12:2393-403.

6. Schaer M, Cuadra MB, Tamarit L, et al. A surface-based approach to quantify local cortical gyrification. IEEE Trans Med Imaging 2008; 27:161-70. 
7. Klyachko VA, Stevens CF. Connectivity optimization and the positioning of cortical areas. Proc Natl Acad Sci 2003;100:7937-41.

8. Borrell V. How cells fold the cerebral cortex. J Neurosci 2018;38:776-83.

9. Kroenke CD, Bayly PV. How forces fold the cerebral cortex. J Neurosci 2018;38:767-75.

10. Fernández V, Llinares-Benadero C, Borrell V. Cerebral cortex expansion and folding: what have we learned? EMBO J 2016;35: 1021-44.

11. Schaer M, Eliez S. Contribution of structural brain imaging to our understanding of cortical development process. Eur Psychiatry Rev 2009;2:13-6.

12. Armstrong E, Schleicher A, Omran H, et al. The ontogeny of human gyrification. Cereb Cortex N Y N 1991 1995;5:56-63.

13. Zwicker A, Denovan-Wright EM, Uher R. Gene-environment interplay in the etiology of psychosis. Psychol Med 2018;1-12.

14. Nanda P, Tandon N, Mathew IT, et al. Local gyrification index in probands with psychotic disorders and their first-degree relatives. Biol Psychiatry 2014;76:447-55.

15. Nesvåg R, Schaer M, Haukvik UK, et al. Reduced brain cortical folding in schizophrenia revealed in two independent samples. Schizophr Res 2014;152:333-8.

16. Falkai P, Honer WG, Kamer T, et al. Disturbed frontal gyrification within families affected with schizophrenia. J Psychiatr Res 2007; 41:805-13.

17. Liu B, Zhang X, Cui Y, et al. Polygenic risk for schizophrenia influences cortical gyrification in 2 independent general populations. Schizophr Bull 2017;43:673-80.

18. Weinberger DR. Schizophrenia and the frontal lobe. Trends Neurosci 1988;11:367-70.

19. Kulynych JJ, Luevano LF, Jones DW, et al. Cortical abnormality in schizophrenia: an in vivo application of the gyrification index. Biol Psychiatry 1997;41:995-9.

20. Palaniyappan L, Mallikarjun P, Joseph V, et al. Folding of the prefrontal cortex in schizophrenia: regional differences in gyrification. Biol Psychiatry 2011;69:974-9.

21. Kelly, S, Jahanshad, N, Zalesky, A, et al. Widespread white matter microstructural differences in schizophrenia across 4322 individuals: results from the ENIGMA Schizophrenia DTI Working Group. Mol Psychiatry 2018;23:1261-69.

22. Selemon LD, Kleinman JE, Herman MM, et al. Smaller frontal gray matter volume in postmortem schizophrenic brains. Am J Psychiatry 2002;159:1983-91.

23. Palaniyappan L, Marques TR, Taylor H, et al. Cortical folding defects as markers of poor treatment response in first-episode psychosis. JAMA Psychiatry 2013;70:1031-40.

24. Guo S, Iwabuchi S, Balain V, et al. Cortical folding and the potential for prognostic neuroimaging in schizophrenia. Br J Psychiatry J Ment Sci 2015;207:458-9.

25. Fusar-Poli P, Bonoldi I, Yung AR, et al. Predicting psychosis: metaanalysis of transition outcomes in individuals at high clinical risk. Arch Gen Psychiatry 2012;69:220-9.

26. Uher R, Cumby J, MacKenzie LE, et al. A familial risk enriched cohort as a platform for testing early interventions to prevent severe mental illness. BMC Psychiatry 2014;14:344.

27. Endicott J, Spitzer RL. A diagnostic interview: the Schedule for Affective Disorders and Schizophrenia. Arch Gen Psychiatry 1978;35: 837-44.

28. First MB. Structured Clinical Interview for the DSM (SCID). Hoboken (NJ): John Wiley and Sons; 2015. Available: https://onlinelibrary. wiley.com/doi/10.1002/9781118625392.wbecp351 (accessed 2019 Oct. 28).
29. Kaufman J, Birmaher B, Brent D, et al. Schedule for Affective Disorders and Schizophrenia for School-Age Children-Present and Lifetime Version (K-SADS-PL): initial reliability and validity data. J Am Acad Child Adolesc Psychiatry 1997;36:980-8.

30. Wechsler D. Wechsler Abbreviated Scale of Intelligence. 2nd ed. San Antonio (TX): NCS Pearson; 2011.

31. Miller TJ, McGlashan TH, Rosen JL, et al. Prodromal assessment with the structured interview for prodromal syndromes and the scale of prodromal symptoms: predictive validity, interrater reliability, and training to reliability. Schizophr Bull 2003;29:703-15.

32. Arseneault L, Cannon M, Fisher HL, et al. Childhood trauma and children's emerging psychotic symptoms: a genetically sensitive longitudinal cohort study. Am J Psychiatry 2011;168:65-72.

33. Poulton R, Caspi A, Moffitt TE, et al. Children's self-reported psychotic symptoms and adult schizophreniform disorder: a 15-year longitudinal study. Arch Gen Psychiatry 2000;57:1053-8.

34. Polanczyk G, Moffitt TE, Arseneault L, et al. Etiological and clinical features of childhood psychotic symptoms: results from a birth cohort. Arch Gen Psychiatry 2010;67:328-38.

35. Fux L, Walger P, Schimmelmann BG, et al. The Schizophrenia Proneness Instrument, Child and Youth version (SPI-CY): practicability and discriminative validity. Schizophr Res 2013;146:69-78

36. Klosterkötter J, Hellmich M, Steinmeyer EM, et al. Diagnosing schizophrenia in the initial prodromal phase. Arch Gen Psychiatry 2001;58:158-64.

37. Schultze-Lutter F, Ruhrmann S, Fusar-Poli P, et al. Basic symptoms and the prediction of first-episode psychosis. Curr Pharm Des 2012;18:351-7.

38. MacKenzie LE, Abidi S, Fisher HL, et al. Stimulant medication and psychotic symptoms in offspring of parents with mental illness. Pediatrics 2016;137:1-10.

39. MacKenzie LE, Patterson VC, Zwicker A, et al. Hot and cold executive functions in youth with psychotic symptoms. Psychol Med 2017;47:2844-53.

40. Glasser MF, Sotiropoulos SN, Wilson JA, et al. The minimal preprocessing pipelines for the Human Connectome Project. NeuroImage 2013;80:105-24

41. Fischl B, van der Kouwe A, Destrieux C, et al. Automatically parcellating the human cerebral cortex. Cereb Cortex 2004;14:11-22.

42. Desikan RS, Ségonne F, Fischl B, et al. An automated labeling system for subdividing the human cerebral cortex on MRI scans into gyral based regions of interest. NeuroImage 2006;31:968-80.

43. R Core Team. R: a language and environment for statistical computing. Vienna: R Foundation for Statistical Computing; 2013. Available: www.R-project.org/ (accessed 2019 Oct. 28).

44. Benjamini Y, Hochberg Y. Controlling the false discovery rate: a practical and powerful approach to multiple testing. J $R$ Stat Soc Ser B Methodol 1995;57:289-300.

45. Bartholomeusz CF, Whittle SL, Montague A, et al. Sulcogyral patterns and morphological abnormalities of the orbitofrontal cortex in psychosis. Prog Neuropsychopharmacol Biol Psychiatry 2013; 44:168-77.

46. Takayanagi Y, Takahashi T, Orikabe L, et al. Volume reduction and altered sulco-gyral pattern of the orbitofrontal cortex in firstepisode schizophrenia. Schizophr Res 2010;121:55-65.

47. Isomura S, Hashimoto R, Nakamura M, et al. Altered sulcogyra patterns of orbitofrontal cortex in a large cohort of patients with schizophrenia. N P J Schizophr 2017;3:3.

48. Pantelis C, Velakoulis D, McGorry PD, et al. Neuroanatomical abnormalities before and after onset of psychosis: a cross-sectional and longitudinal MRI comparison. Lancet 2003;361:281-8. 
49. Cannon TD, Chung $\mathrm{Y}, \mathrm{He}$ G, et al. Progressive reduction in cortical thickness as psychosis develops: a multisite longitudinal neuroimaging study of youth at elevated clinical risk. Biol Psychiatry 2015;77:147-57.

50. Homayoun H, Moghaddam B. Orbitofrontal cortex neurons as a common target for classic and glutamatergic antipsychotic drugs. Proc Natl Acad Sci U S A 2008;105:18041-6.

51. Rolls ET, Grabenhorst F. The orbitofrontal cortex and beyond: from affect to decision-making. Prog Neurobiol 2008;86:216-44.

52. Seo H, Lee D. Neural basis of learning and preference during social decision-making. Curr Opin Neurobiol 2012;22:990-5.

53. Palaniyappan L, Liddle PF. Diagnostic discontinuity in psychosis: a combined study of cortical gyrification and functional connectivity. Schizophr Bull 2014;40:675-84.

54. Chang, M, Womer, FY, Bai, C, et al. Voxel-based morphometry in individuals at genetic high risk for schizophrenia and patients with schizophrenia during their first episode of psychosis. PLoS One 2016;11:e0163749.

55. Schultz CC, Wagner G, Koch K, et al. The visual cortex in schizophrenia: alterations of gyrification rather than cortical thickness-a combined cortical shape analysis. Brain S truct Funct 2013;218:51-8.

56. Kong L, Herold CJ, Lässer MM, et al. Association of cortical thickness and neurological soft signs in patients with chronic schizophrenia and healthy controls. Neuropsychobiology Basel 2015;71:225-33.

57. Thompson CA, Karelis J, Middleton FA, et al. Associations between neurodevelopmental genes, neuroanatomy, and ultra high risk symptoms of psychosis in 22q11.2 deletion syndrome. Am J Med Genet B Neuropsychiatr Genet 2017;174:295-314.
58. Walton E, Geisler D, Hass J, et al. The impact of genome-wide supported schizophrenia risk variants in the neurogranin gene on brain structure and function. PLOS ONE 2013;8:e76815.

59. Sasabayashi D, Takayanagi Y, Takahashi T, et al. Increased occipital gyrification and development of psychotic disorders in individuals with an at-risk mental state: a multicenter study. Biol Psychiatry 2017;82:737-45

60. Shollenbarger SG, Price J, Wieser J, et al. Impact of cannabis use on prefrontal and parietal cortex gyrification and surface area in adolescents and emerging adults. Dev Cogn Neurosci 2015; 16:46-53.

61. Spencer TJ, Brown A, Seidman LJ, et al. Effect of psychostimulants on brain structure and function in ADHD: a qualitative literature review of MRI-based neuroimaging studies. J Clin Psychiatry 2013; 74:902-17.

62. Bagot KS, Milin R, Kaminer Y. Adolescent initiation of cannabis use and early-onset psychosis. Subst Abuse 2015;36:524-33.

63. Haijma SV, Van Haren N, Cahn W, et al. Brain volumes in schizophrenia: a meta-analysis in over 18000 subjects. Schizophr Bull 2013;39:1129-38.

64. Buckner RL, Head D, Parker J, et al. A unified approach for morphometric and functional data analysis in young, old, and demented adults using automated atlas-based head size normalization: reliability and validation against manual measurement of total intracranial volume. NeuroImage 2004;23:724-38.

65. Janssen J, Alemán-Gómez Y, Schnack H, et al. Cortical morphology of adolescents with bipolar disorder and with schizophrenia. Schizophr Res 2014;158:91-9.

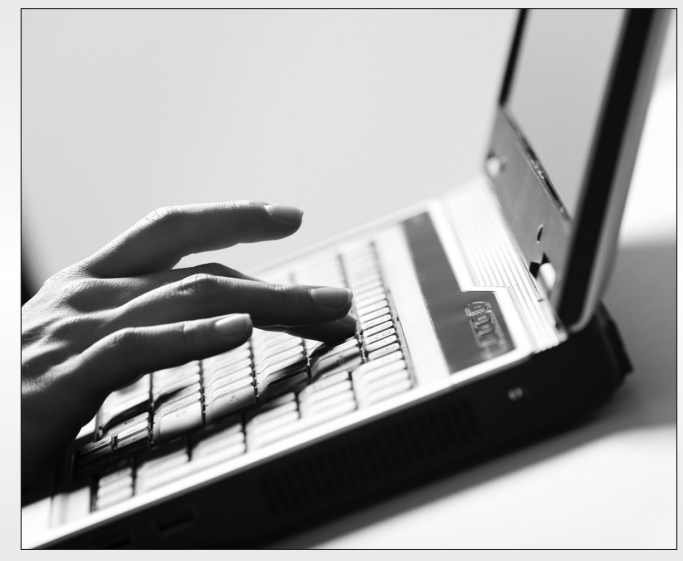

Journal of Psychiatry e) Neuroscience

\author{
Online manuscript \\ submission and peer review
}

AVAILABLE at

http://mc.manuscriptcentral.com/jpn 